\title{
Comparison of Knee Muscle Strength and Ankle Dorsiflexion Range of Motion Between Standing Workers With and Without Patellofemoral Pain Syndrome
}

\author{
Young-soo Weon ${ }^{1,2}$, BPT, PT, Sun-hee Ahn' ${ }^{2,3}, \mathrm{PhD}, \mathrm{PT}$, Jun-hee Kim²,3, PhD, PT, Gyeong-tae Gwak ${ }^{1,2}, \mathrm{BPT}, \mathrm{PT}$, \\ Oh-yun Kwon ${ }^{2,3}$, PhD, PT \\ ${ }^{1}$ Department of Physical Therapy, The Graduate School, Yonsei University, ${ }^{2}$ Kinetic Ergocise Based on Movement Analysis Laboratory, \\ ${ }^{3}$ Department of Physical Therapy, College of Health Science, Yonsei University, Wonju, Korea
}

\author{
Article Info \\ Received October 13, 2020 \\ Revised October 27, 2020 \\ Accepted October 28, 2020 \\ Corresponding Author \\ Oh-yun Kwon \\ E-mail: kwonoy@yonsei.ac.kr \\ https://orcid.org/0000-0002-9699-768X
}

\section{Key Words}

Hamstring muscles

Knee joint

Patellofemoral pain syndrome

Quadriceps muscle
Background: Prolonged standing during work causes a lower extremity pain and disorders. Patellofemoral pain syndrome (PFPS) is one of the common diagnoses of the knee pain. Although the etiology of PFPS is not completely understood, it is considered to be multifactorial.

Objects: The purpose of this study was to investigate difference in strength of knee muscles, quadriceps:hamstring muscles strength ratio ( $\mathrm{Q}: \mathrm{H}$ ratio), asymmetry ratio of knee muscles strength and dorsiflexion range of motion (ROM) between standing workers with and without PFPS.

Methods: Twenty-eight standing workers with PFPS and 26 age-, height-, and weightmatched standing workers without PFPS participated in this study. A tension sensor measured knee muscle strength, and motion sensor measured dorsiflexion ROM. The asymmetry ratio of knee muscles was calculated by a specific formula using the knee muscles strength of the dominant side and the sound side. An independent t-test was used to identify significant differences in the strength, ROM, Q:H ratio, and asymmetry ratio between the PFPS and normal groups.

Results: The standing worker with PFPS have significantly lower dorsiflexion ROM ( $p<$ $0.000)$ and higher asymmetry ratio of the hamstring muscles strength $(p<0.000)$ compare to the standing worker without PFPS. No significant differences were seen in the strength of quadriceps muscle and hamstring muscles, Q:H ratio, and asymmetry ratio of quadriceps muscle strength.

Conclusion: There was a significant difference in the asymmetry ratio of the isometric hamstring muscle strength. This finding suggests that the asymmetry ratio of isometric hamstring muscle strength may be more important than measuring only the hamstring muscle strength of the PFPS side. Furthermore, the results of this study showed a significant difference in dorsiflexion ROM between the standing industrial workers with and without PFPS. Dorsiflexion ROM and isometric hamstring muscle strength should be considered when evaluating the subjects with PFPS.

\section{INTRODUCTION}

Standing is considered a versatile and effective position for many process-related jobs in industrial workplaces due to the increased mobility and degree of freedom of the legs. However, when workers spend long periods of time in the standing position during their working hours, they may feel discomfort and experience muscle fatigue by the end of the workday [1]. Prolonged standing or walking can increase the duration of compressive forces, exacerbate fatigue, and lead to damage in the leg and foot tissues [2,3]. Prolonged work-related risk factor could lead to progressive abnormal biomechanical function and result in a greater risk of chronic lower extremity musculoskeletal symptoms and malalignment [4,5].

Knee pain is a common musculoskeletal symptom among people of working age. Epidemiological studies have reported that prolonged standing at work is associated with lowerextremity pain and discomfort [6,7]. Patellofemoral pain syndrome (PFPS) is one of the common diagnoses of knee pain [8]. Individuals with PFPS often report pain in the retro patellar or 
peri patellar region during tasks that increase patellofemoral joint loading [9], likely due to altered tracking or increased stress in the lateral patellofemoral joint [10]. Although the etiology of PFPS is not completely understood, it is considered to be multifactorial [11]. A complex two-way interaction of kinematics and kinetics exists in the lower extremities, which indicates that the kinematics and kinetics around the knee joint are affected by distal and proximal factors [12]. This implies that foot, knee, and hip alignment may all be important in the pathogenesis of PFPS $[13,14]$.

Impaired muscle function is often found in patients with PFPS. The quadriceps and hamstrings muscles are the largest contributors to articular loading on the knee during walking and impact the distribution of load across the knee $[15,16]$. The quadriceps muscle is often weakened in patients with PFPS, and the decreased quadriceps torque, especially with eccentric knee extension, is found to be associated with PFPS $[17,18]$. Additionally, muscle strength imbalances between the quadriceps and hamstring muscles are evident in patients with PFPS [19]. This muscle imbalance usually reflects weakened quadriceps muscles but normal strength of the hamstring muscles, which subsequently results in a lower quadriceps:hamstring muscles (Q:H) ratio [20].

Tightness of soft tissues, such as the gastrocnemius, have also been suggested to influence PFPS. Lack of flexibility of the gastrocnemius and soleus complex is associated with limited ankle dorsiflexion [21]. Lack of ankle dorsiflexion during gait may result in excessive subtalar joint pronation and tibial internal rotation to gain additional range of motion (ROM) for the terminal stance phase of gait [22]. Excessive tibial internal rotation leads to an increased Q-angle and femoral internal rotation and consequently increased patellofemoral stresses [23]. However, no studies have compared the knee muscle strength, Q:H ratio, asymmetry ratio of knee muscle, and ankle ROM between standing industrial workers with and without PFPS. The main purpose of this study was therefore to investigate the differences in the quadriceps muscle strength, hamstring muscle strength, Q:H strength ratio, asymmetry ratio of knee muscle strength, and dorsiflexion ROM between standing industrial workers with and without PFPS.

\section{MATERIALS AND METHODS}

\section{Subjects}

Fifty-four male standing industrial workers (mean age \pm standard deviation [SD], $44.69 \pm 7.92$ years; range, 31-59 years) who worked for forty a week were recruited from an automobile assembly factory. Twenty-eight standing industrial workers with PFPS and 26 age-, height-, and weight-matched standing industrial workers without PFPS participated in this study. All the subjects were provided with an explanation of the risks and benefits associated with the study and signed an informed consent form. Workers with PFPS were included if they had a positive sign in two or more PFPS screening tests and in two of the specific PFPS tests. The PFPS screening and specific PFPS tests were conducted by an inspector, with 5 years' experience as a musculoskeletal practitioner. The PFPS screening tests were (a) vague or localized patella or retropatella pain for $>3$ months [23]; (b) pain $>3 / 10$ on a numeric rating scale reproduced with running [24] and at least one of the following [23]: stair descent or kneeling or squatting or prolonged sitting. The specific PFPS tests used in this study were the patellar apprehension test [25] and Clarke's test [26]. The exclusion criteria were a history of dislocation or fracture of the patella [23], and lower limb surgery within the previous 4 months [27]. This study was approved by the Yonsei University Mirea Institutional Review Board (approval No. 1041849202007-BM-078-01).

\section{Experimental Instruments}

\section{1) Smart KEMA motion sensor}

A Smart KEMA motion sensor (KOREATECH Co., Ltd., Seoul, Korea) was used to measure the kinematics of dorsiflexion. The unit of the Smart KEMA motion sensor output is degrees for all ROM measurements. The motion sensors were mounted on a plastic frame and placed in a Smart KEMA motion strap (KOREATECH Co., Ltd.) to fix the motion sensors in position. The motion sensors contained a tri-axillar gyroscope, a magnetometer, and an accelerometer, as well as a signal converter and a signal transmission sensor. The motion sensor data were transmitted to a recording Android tablet with Smart KEMA software, using a $25 \mathrm{~Hz}$ sampling frequency. The value of the real-time angle for 5 seconds was recorded at the end of the ROM, and the Smart KEMA software computed the average of the angle data for the middle 3 seconds for the data analysis. 


\section{2) Smart KEMA tension sensor}

The isometric muscle strength of knee flexion and extension were measured using a Smart KEMA tension sensor (KOREATECH Co., Ltd.). The tension sensor contained a load cell that had a measurement range of $0-1,960 \mathrm{~N}$ with an accuracy of \pm 4.9 N. The tension sensor data were transmitted at a sampling frequency of $10 \mathrm{~Hz}$ to a recording Android tablet that was running the Smart KEMA software and computed the average of the data for the middle 3 seconds for the data analysis. In previous studies, a tension sensor was used to measure isometric strength, and this showed high intra-rater reliability [28]. The tension sensor had two rings. One side was fixed to a glass suction cup or a stable material using an orthopedic belt and the other side was fixed to a body segment using the Smart KEMA tension strap (KOREATECH Co., Ltd.).

\section{Outcome Measures}

\section{1) Dorsiflexion ROM}

To measure ankle dorsiflexion ROM, each subject was required to lie in the prone position with the knee extended and the foot positioned beyond the edge of the plinth. The Smart KEMA motion sensor was fixed to the lateral aspect of the subject's fifth metatarsal using the Smart KEMA motion strap. To prevent compensation such as pronation of the subtalar joint, the examiner neutralized the subtalar joint. The examiner held the subject's metatarsals and dorsiflexion direction until resistance was felt. The dorsiflexion ROM was measured when the examiner felt the end-feel of the ankle joint (Figure 1).

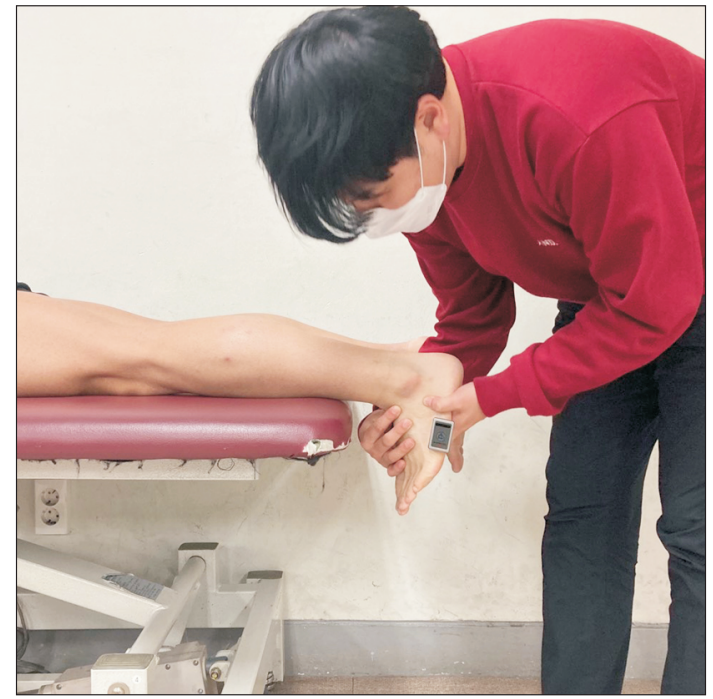

Figure 1. Dorsiflexion range of motion.

\section{2) Quadriceps muscle strength}

To measure the isometric strength of the quadriceps muscles, the length of the restraining belt was adjusted so that the subjects could reach a knee extension of $45^{\circ}$. The subjects performed knee extensions against a strap anchored by the therapeutic table to measure the maximal voluntary isometric contraction (MVIC) twice (Figure 2). An ankle strap was placed on the ankle above the lateral malleolus. The subjects were shown how to stabilize themselves by holding onto the side of the table with their hands while sitting upright. To prevent compensation such as posterior of the pelvis, the examiner fixed the pelvis (Figure 3). Strength was analyzed by averaging the middle 3 seconds of 5 seconds measurement. Strength was

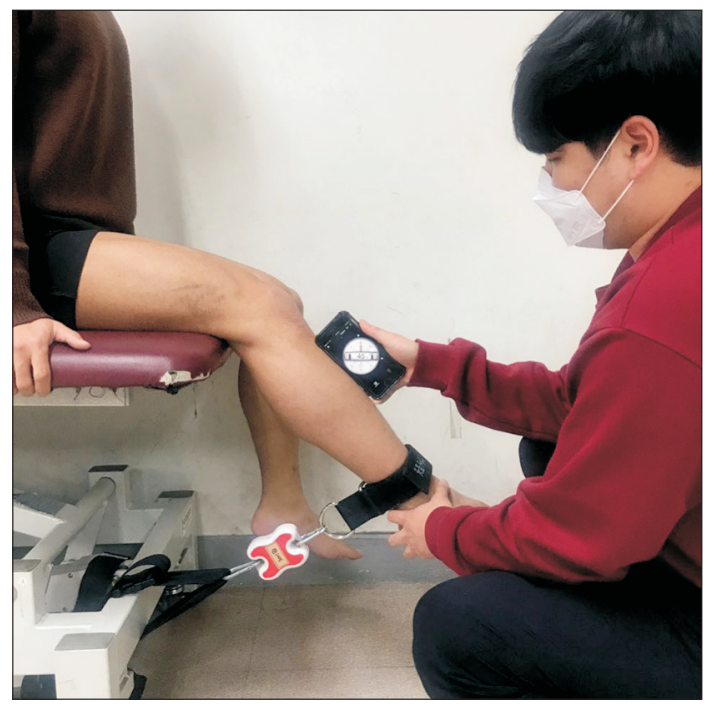

Figure 2. Quadriceps muscle strength.

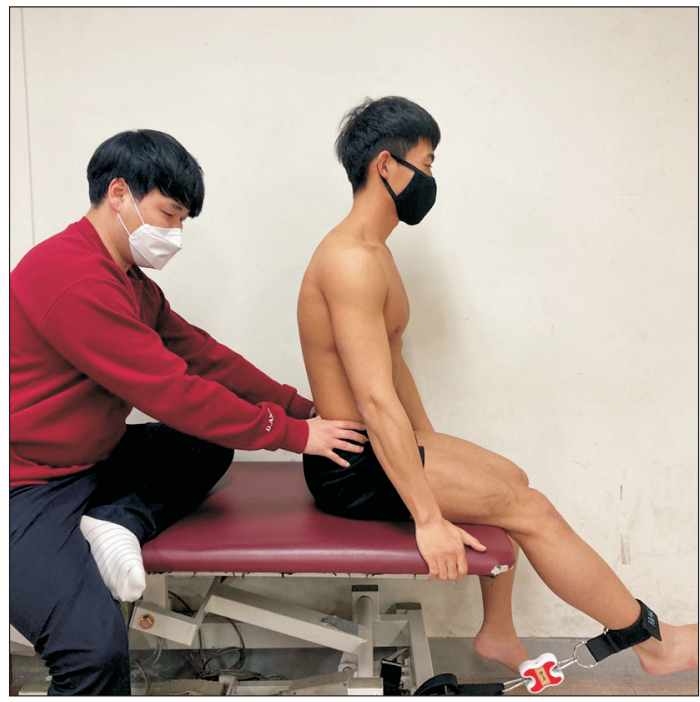

Figure 3. Quadriceps muscle strength. 
normalized by the subject's body weight (N/kg). Strength was measured two times and averaged for the data analysis.

\section{3) Hamstring muscle strength}

The isometric strength measurement of the hamstring muscles was performed with the subject in the prone position. The subject flexed the knee at $45^{\circ}$. The knee flexion was performed against a strap anchored by a glass suction cup to measure the MVIC twice. An ankle strap was placed on the ankle above the lateral malleolus. To prevent compensation such as anterior of the pelvis, the examiner fixed the pelvis (Figure 4). Strength was analyzed by averaging the middle 3 seconds of 5 seconds measurement. Strength was normalized by body weight $(\mathrm{N} / \mathrm{kg})$, measured two times, and averaged for the data analysis.

\section{4) Asymmetry ratio}

The asymmetry ratio between the sound and PFPS sides was calculated using the following formula: percent asymmetry ratio of the strength of the quadriceps and hamstring muscles $=($ sound side - PFPS side $) /($ sound side + PFPS side $) \times 100$. To calculate the percent asymmetry ratio of knee muscle strength in the standing workers without PFPS, the dominant side was replaced with the sound side in the asymmetry ratio formula when comparing the normal worker group to the PFPS group with a sound side, and the non-dominant side was replaced with the PFPS side when comparing the normal worker group to the PFPS group with a PFPS side [29,30].

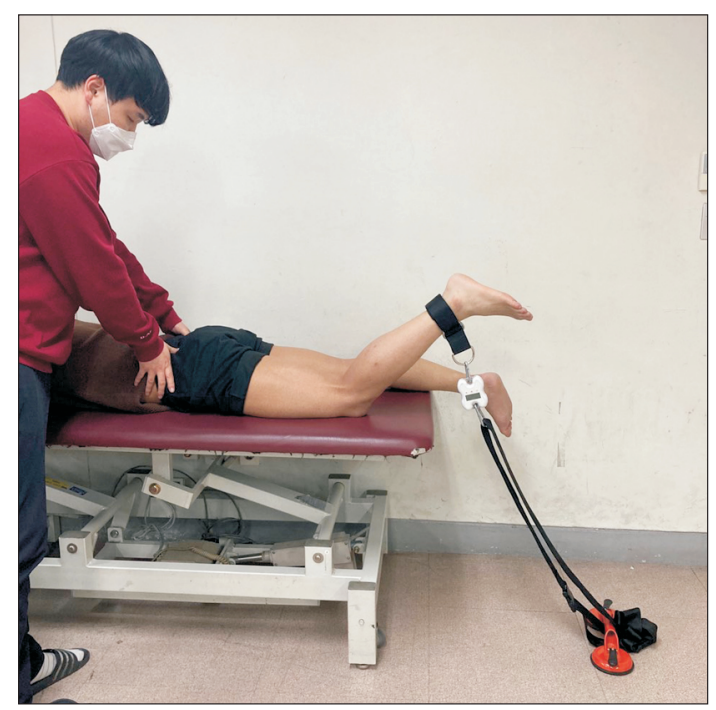

Figure 4. Hamstring muscles strength.

\section{Experimental Procedures}

Before data collection, the subjects were familiarized with the testing protocol. Provided instructions, and asked to practice the dorsiflexion ROM and knee strength measurement to ensure proper test. Then, dorsiflexion ROM and knee muscle strength (quadriceps, hamstrings) was measured in random order. The random order was determined by drawing lots. Mean values from two measurements of dorsiflexion ROM and knee strength were used for data analysis.

\section{Data Collection}

The data from the Smart KEMA motion sensor and Smart KEMA tension sensor were recorded at sampling frequencies of $25 \mathrm{~Hz}$ and $10 \mathrm{~Hz}$, respectively, and transmitted to an Android tablet over a Bluetooth connection using the Smart KEMA application software (KOREATECH Co., Ltd.). The dorsiflexion ROM data were expressed in degrees and the knee muscle strength measurement data were expressed in kilograms. The collected knee muscle strength data were normalized to body weight. The normalized strength was represented as a percentage of body weight, where percentage of body weight $=($ maximal strength of each trial $[\mathrm{kg}] /$ body weight $[\mathrm{kg}]) \times 100$ [31]. The average strength values of the two measurements were calculated for subsequent analyses.

\section{Statistical Analysis}

In this study, the data were expressed as mean \pm SD. All the data were tested for normal distribution using the KolmogorovSmirnov normality test. An independent t-test was used to identify significant differences in the strength, ROM, Q:H ratio, and asymmetry ratio between the PFPS and normal groups. All the analyses were performed using SPSS 25.0 (IBM Corp., Armonk, NY, USA). The level of significance was set at $p=0.05$.

\section{RESULTS}

Table 1 summarizes the characteristics of the participants in each group at baseline. The mean age of the participants was nearly 45 years with a range of 31-59 years. Independent t-tests were performed on the baseline outcome measures, and there was no significant differences in the age, height and weight of the participants between the groups. 
Table 1. Characteristics of the workers with and without PFPS

\begin{tabular}{|c|c|c|c|c|}
\hline Characteristics & Total ( $N=54)$ & Workers with PFPS ( $\mathrm{n}=28$ ) & Workers without PFPS $(n=26)$ & $\mathrm{p}$-value \\
\hline Age lyl & $44.69 \pm 7.92$ & $44.86 \pm 7.64$ & $44.50 \pm 8.36$ & 0.596 \\
\hline Body height $(\mathrm{cm})$ & $171.50 \pm 4.60$ & $171.68 \pm 5.15$ & $171.31 \pm 4.03$ & 0.161 \\
\hline Body weight (kg) & $73.17 \pm 9.47$ & $73.50 \pm 10.06$ & $72.81 \pm 8.97$ & 0.261 \\
\hline
\end{tabular}

Values are presented as mean \pm standard deviation. PFPS, patellofemoral pain syndrome.

Table 2. Comparison of the dorsiflexion ROM, knee muscle strength, and Q:H ratio between the workers with and without PFPS

\begin{tabular}{lccc}
\hline & Workers with PFPS $(\mathrm{n}=28)$ & Workers without PFPS $(\mathrm{n}=26)$ & $\mathrm{p}$-value \\
\hline Dorsiflexion $\mathrm{ROM}\left({ }^{\circ}\right)$ & $9.52 \pm 3.17$ & $13.45 \pm 3.38$ & $<0.000^{*}$ \\
Quadriceps muscle strength $\left(\mathrm{kgf} \cdot \mathrm{BW}^{-1}\right)$ & $44.04 \pm 15.69$ & $47.81 \pm 17.74$ & 0.413 \\
Hamstring muscles strength $\left(\mathrm{kgf} \cdot \mathrm{BW}^{-1}\right)$ & $23.05 \pm 9.12$ & $18.77 \pm 6.67$ & 0.402 \\
Q:H ratio & $1.97 \pm 0.07$ & $2.19 \pm 0.13$ & 0.152
\end{tabular}

Values are presented as mean \pm standard deviation. ROM, range of motion; $Q: H$, quadriceps muscles:hamstring muscles; PFPS, patellofemoral pain syndrome. ${ }^{*} \mathrm{p}<0.05$.

Table 3. Comparison of the asymmetry ratio of knee strength between the workers with and without PFPS

\begin{tabular}{lccc} 
& Workers with PFPS $(\mathrm{n}=28)$ & Workers without PFPS (n = 26) & $p$-value \\
\hline Hamstring muscles asymmetry ratio (\%) & $-9.50 \pm 6.43$ & $5.32 \pm 4.01$ & $<0.000^{*}$ \\
Quadriceps muscle asymmetry ratio (\%) & $5.42 \pm 4.81$ & $6.61 \pm 5.44$ & 0.402 \\
\hline
\end{tabular}

Values are presented as mean \pm standard deviation. PFPS, patellofemoral pain syndrome. ${ }^{*} p<0.05$.

\section{Dorsiflexion ROM and Strength of Knee Muscles}

The results of the independent t-test indicated that the workers with PFPS had a significantly lower dorsiflexion ROM than the workers without PFPS $(p<0.05)$. However, there was no significant difference in the isometric muscle strength of the quadriceps and hamstring muscles and the Q:H ratio between the workers with and without PFPS ( $p>0.05$ ) (Table 2).

\section{Asymmetry of Knee Muscles Strength}

The asymmetry ratios of the isometric muscle strength of the hamstring muscles was significantly higher in the workers with PFPS compared to those without PFPS ( $p<0.05)$. However, there were no significant differences in the asymmetry ratio of isometric muscle strength of the quadriceps muscles between the two groups $(p>0.05)$ (Table 3).

\section{DISCUSSION}

The primary purpose of this study was to compare the knee joint muscle strength, ankle joint dorsiflexion ROM, and asymmetry ratio of the knee muscles strength between standing workers with and without PFPS. The standing worker with PFPS demonstrated significantly lower dorsiflexion ROM and a significantly higher asymmetry ratio of isometric hamstring muscles strength. However, there were no differences in the isometric strength of the quadriceps and hamstring muscles, isometric Q:H strength and asymmetry ratio of isometric quadriceps muscle strength.

Similarly to our results, a study by Rabin et al. found a reduction in dorsiflexion ROM among individuals with PFPS compared to the asymptomatic group [32]. The results of a study by Arndt et al. were also consistent with those of our study. Arndt et al reported a maximum of $18.7^{\circ}$ of dorsiflexion during gait on a flat surface [33]. Therefore, if patients with PFPS have insufficient dorsiflexion ROM, they may use compensatory mechanisms such as excessive subtalar joint pronation and consequently increased external rotation of the tibia, or perhaps femur internal rotation, to gain additional motion during terminal stance phase of gait [23]. Stergio et al. [34] suggested that tight plantar flexors and increased subtalar joint pronation could increase ground reaction impact forces and lateral deviation of the tibia as a rebound effect due to the increased impact with the ground during gait and may be a source of various injuries. In contrast, Mølgaard et al. [35] reported a significantly larger dorsiflexion ROM in the PFPS group $\left(22.28^{\circ}\right.$ vs. $\left.17.78^{\circ}\right)$ in their study, and Powers et al. [36] demonstrated greater ankle dorsiflexion in patients with PFPS compared with the control group when the patients were 
walking fast, descending stairs, and descending ramps. The reason the results of these studies are contrary to ours may be the difference in measurement methods. We measured the dorsiflexion ROM with neutralization of the subtalar joint, while they measured the dorsiflexion ROM without subtalar joint neutralization. Moreover, we measured the dorsiflexion ROM in a non-weight-bearing condition, but they measured it in a weight-bearing condition. Compensation such as subtalar joint pronation may therefore have occurred when measuring the dorsiflexion ROM in their studies.

Quadriceps muscle weakness, especially in eccentric strength on the painful side compared to the uninvolved side, is seen in PFPS [17,19,37]. Supporting this, Duvigneaud et al. [38] and Boling et al. [39] discovered that military recruits who later developed PFP had, 10\% lower isokinetic knee extensor and knee flexion peak torque than the recruits who did not develop PFPS during basic military training. However, the present study did not find a significant difference in the isometric strength of the quadriceps and hamstring muscles. We measured muscle strength by isometric contraction, and our results were consistent with those of several studies [40,41]. Willson and Davis [40] demonstrated that there was no difference in the isometric strength of the knee muscles between subjects with and without PFPS, and the magnitude of the differences in strength between the groups for knee flexion and extension strength was neither clinically nor statistically significant. Rathleff et al. [41] reported that, in their study, patellofemoral pain did not reflect decreased isometric muscle strength of the knee muscles among age- and sex-matched pain-free adolescents. This result shows that measuring isokinetic knee extension strength is more important than evaluating the isometric knee extension strength of PFPS patients.

Although there was no difference in the isometric strength of the hamstring muscles between the PFPS and non-PFPS groups in our study, there was a significant difference in the asymmetry ratio of the isometric hamstring muscle strength. Although the asymmetry ratio of the standing workers with PFPS was negative $(-9.50 \%)$, it was positive $(+5.32 \%)$ for the control group. These differences indicate that the subjects with PFPS had stronger hamstring muscle strength on the PFPS side than the sound side. Besier et al. [42] showed that the patients with PFPS in their study had greater co-contraction of the quadriceps and hamstring muscles compared to the controls without symptoms of PFPS. In this study, the subjects with
PFPS showed 30\%-50\% higher hamstring muscle force during both walking and running [42]. Moreover, Besier et al. [42] demonstrated that such excessive use of the hamstring muscles in individuals with PFPS may cause greater joint contact forces and joint stresses than among pain-free subjects. These results indicate that the asymmetry ratio of isometric hamstring muscle strength may be more important than measuring only the hamstring muscle strength of the PFPS side.

The Q:H ratio has previously been used not only to examine the similarity between quadriceps and hamstring muscle strength, but also to assess knee functional ability and muscle balance $[43,44]$. The Q:H ratio has conventionally been expressed as concentric quadriceps to concentric hamstring muscle strength $[45,46]$. In previous studies, various methods were used to measure the $\mathrm{Q}: \mathrm{H}$ strength ratio, including concentric quadriceps to concentric hamstring muscle strength, eccentric quadriceps to concentric hamstring muscle strength, and concentric quadriceps to eccentric hamstring muscle strength $[47,48]$. The conventional Q:H ratio in patients with PFPS has been found to be relatively low (1.66 to 2.0) compared to that of healthy subjects (1.33 to 1.53 ) $[19,43,47,49,50]$. In contrast, our results showed no significant difference between the PFPS group $(1.97 \pm 0.07)$ and the control group $(2.19 \pm 0.13)$. There are several possible reasons for this result. The first is the use of a different measurement posture. In other studies, the researchers measured the strength of the quadriceps and hamstring muscles in the same posture using an isokinetic dynamometer, but the measuring posture of the quadriceps and hamstring muscles were different in our study. The second is the difference in measurement methods. We measured the strength of the quadriceps and hamstring muscles as isometric strength, but other researchers measured the muscles' isokinetic strength. These results indicate that it may be more accurate to measure isokinetic strength than isometric strength when measuring the Q:H ratio of PFPS patients. Further research is needed to determine whether there is a difference between PFPS patients and healthy subjects even when measuring isometric hamstring muscle strength in the same posture.

Our study had several limitations. First, our results cannot be generalized because all the subjects were men. Further studies are therefore needed to establish whether the findings apply to female patients with PFPS. Second, we did not obtain electromyographic measurements of the knee muscles when mea- 
suring quadriceps and hamstring muscle strength. Third, the strength of hip external rotation, abduction, and the Q-angle, one of the important risk factors in PFPS, was not measured. Fourth, standing workers participated in our study, but we did not include measurements in closed kinetic chains such as walking, climbing and descending stairs, and squats.

\section{CONCLUSIONS}

Although there was no significant difference in the isometric hamstring muscle strength between the standing workers with and without PFPS in our study, there was a significant difference in the asymmetry ratio of the isometric hamstring muscle strength. This finding suggests that the asymmetry ratio of isometric hamstring muscle strength may be more important than measuring only the hamstring muscle strength of the PFPS side. Furthermore, the results of this study showed a significant difference in dorsiflexion ROM between the standing industrial workers with and without PFPS. Dorsiflexion ROM and isometric hamstring muscle strength should be considered when evaluating the subjects with PFPS.

\section{ACKNOWLEDGEMENTS}

This study was supported by the "Brain Korea 21 FOUR Project", the Korean Research Foundation for Department of Physical Therapy in the Graduate School of Yonsei University.

\section{CONFLICTS OF INTEREST}

No potential conflict of interest relevant to this article was reported.

\section{AUTHOR CONTRIBUTIONS}

Conceptualization: JK, OK. Data curation: YW, JK. Formal analysis: YW, GG. Investigation: YW, JK. Methodology: YW, SA, JK, GG, OK. Project administration: YW, SA, GG. Resources: YW, GG, OK. Supervision: SA, JK, OK. Validation: YW, JK. Visualization: YW. Writing - original draft: YW. Writing - review \& editing: SA, JK, GG, OK.

\section{ORCID}

Young-soo Weon, https://orcid.org/0000-0003-3852-606X

Sun-hee Ahn, https://orcid.org/0000-0001-7660-8598

Jun-hee Kim, https://orcid.org/0000-0002-0498-7207

Gyeong-tae Gwak, https://orcid.org/0000-0003-1812-8910

\section{REFERENCES}

1. Halim I, Omar AR. A review on health effects associated with prolonged standing in the industrial workplaces. Int J Res Rev Appl Sci 2011;8(1):14-21.

2. Reid CR, McCauley Bush P, Karwowski W, Durrani SK. Occupational postural activity and lower extremity discomfort: a review. Int J Ind Ergon 2010;40(3):247-56.

3. Jaffar N, Abdul-Tharim A, Mohd-Kamar I, Lop N. A literature review of ergonomics risk factors in construction industry. Proced Eng 2011;20:89-97.

4. Daneshmandi H, Saki F, Shahheidari S, Khoori A. Lower extremity malalignment and its linear relation with $\mathrm{Q}$ angle in female athletes. Proced-Soc Behav Sci 2011;15:3349-54.

5. Shultz SJ, Nguyen AD, Levine BJ. The relationship between lower extremity alignment characteristics and anterior knee joint laxity. Sports Health 2009;1 (1):54-60.

6. Krijnen RM, de Boer EM, Adèr HJ, Bruynzeel DP. Venous insufficiency in male workers with a standing profession. Part 1: epidemiology. Dermatology 1997;194(2):111-20.

7. Tüchsen F, Hannerz H, Burr H, Krause N. Prolonged standing at work and hospitalisation due to varicose veins: a 12 year prospective study of the Danish population. Occup Environ Med 2005;62(12):847-50.

8. Esculier JF, Roy JS, Bouyer LJ. Psychometric evidence of selfreported questionnaires for patellofemoral pain syndrome: a systematic review. Disabil Rehabil 2013;35(26):2181-90.

9. D'hondt NE, Struijs PA, Kerkhoffs GM, Verheul C, Lysens R, Aufdemkampe G, et al. Orthotic devices for treating patellofemoral pain syndrome. Cochrane Database Syst Rev 2002; (2):CD002267.

10. Dye SF. The pathophysiology of patellofemoral pain: a tissue homeostasis perspective. Clin Orthop Relat Res 2005;(436): 100-10.

11. Lankhorst NE, Bierma-Zeinstra SM, van Middelkoop M. Risk factors for patellofemoral pain syndrome: a systematic review. J Orthop Sports Phys Ther 2012;42(2):81-94. 
12. Csintalan RP, Schulz MM, Woo J, McMahon PJ, Lee TQ. Gender differences in patellofemoral joint biomechanics. Clin Orthop Relat Res 2002;(402):260-9.

13. Goodfellow J, Hungerford DS, Woods C. Patello-femoral joint mechanics and pathology. 2. Chondromalacia patellae. J Bone Joint Surg Br 1976;58(3):291-9.

14. Lee TQ, Yang BY, Sandusky MD, McMahon PJ. The effects of tibial rotation on the patellofemoral joint: assessment of the changes in in situ strain in the peripatellar retinaculum and the patellofemoral contact pressures and areas. J Rehabil Res Dev 2001;38(5):463-9.

15. Kumar D, Manal KT, Rudolph KS. Knee joint loading during gait in healthy controls and individuals with knee osteoarthritis. Osteoarthritis Cartilage 2013;21(2):298-305.

16. Pandy MG, Andriacchi TP. Muscle and joint function in human locomotion. Annu Rev Biomed Eng 2010;12:401-33.

17. Kaya D, Citaker S, Kerimoglu U, Atay OA, Nyland J, Callaghan M, et al. Women with patellofemoral pain syndrome have quadriceps femoris volume and strength deficiency. Knee Surg Sports Traumatol Arthrosc 2011;19(2):242-7.

18. Dvir Z, Halperin N, Shklar A, Robinson D. Quadriceps function and patellofemoral pain syndrome. Part I: pain provocation during concentric and eccentric isokinetic activity. Isokinet Exerc Sci 1991;1(1):26-30.

19. Werner S. An evaluation of knee extensor and knee flexor torques and EMGs in patients with patellofemoral pain syndrome in comparison with matched controls. Knee Surg Sports Traumatol Arthrosc 1995;3(2):89-94.

20. Brown CA, Carragee C, Sox-Harris A, Merchant AC, Mcadams TR. Prevalence of abnormal patellofemoral congruence in elite American football players and association with quadriceps isokinetic testing. J Knee Surg 2014;27(1):47-52.

21. Benetti MC, Marchese T. Management of common musculoskeletal disorders. J Nurse-Midwifery 1996;41(2):173-87.

22. Leardini A, Stagni R, O'Connor JJ. Mobility of the subtalar joint in the intact ankle complex. J Biomech 2001;34(6):805-9.

23. Piva SR, Goodnite EA, Childs JD. Strength around the hip and flexibility of soft tissues in individuals with and without patellofemoral pain syndrome. J Orthop Sports Phys Ther 2005;35(12):793-801.

24. Farrar JT, Young JP Jr, LaMoreaux L, Werth JL, Poole RM. Clinical importance of changes in chronic pain intensity measured on an 11-point numerical pain rating scale. Pain 2001;94(2):149-58
25. Reider B. The orthopaedic physical examination. Philadelphia: Elsevier Saunders; 2005.

26. Hyde TE, Gengenbach MS. Conservative management of sports injuries. Sudbury: Jones and Bartlett; 2007.

27. Mizner RL, Chmielewski TL, Toepke JJ, Tofte KB. Comparison of 2-dimensional measurement techniques for predicting knee angle and moment during a drop vertical jump. Clin J Sport Med 2012;22(3):221-7.

28. Kim HA, Hwang UJ, Jung SH, Ahn SH, Kim JH, Kwon OY. Comparison of shoulder strength in males with and without myofascial trigger points in the upper trapezius. Clin Biomech (Bristol, Avon) 2017;49:134-8.

29. Moraes GF, Faria CD, Teixeira-Salmela LF. Scapular muscle recruitment patterns and isokinetic strength ratios of the shoulder rotator muscles in individuals with and without impingement syndrome. J Shoulder Elbow Surg 2008;17(1 Suppl):48S-53S.

30. Warner JJ, Micheli LJ, Arslanian LE, Kennedy J, Kennedy R. Patterns of flexibility, laxity, and strength in normal shoulders and shoulders with instability and impingement. Am J Sports Med 1990;18(4):366-75.

31. Hurd WJ, Morrey BF, Kaufman KR. The effects of anthropometric scaling parameters on normalized muscle strength in uninjured baseball pitchers. J Sport Rehabil 2011;20(3):31120.

32. Rabin A, Kozol Z. Measures of range of motion and strength among healthy women with differing quality of lower extremity movement during the lateral step-down test. J Orthop Sports Phys Ther 2010;40(12):792-800.

33. Arndt A, Westblad P, Winson I, Hashimoto T, Lundberg A. Ankle and subtalar kinematics measured with intracortical pins during the stance phase of walking. Foot Ankle Int 2004; 25(5):357-64.

34. Stergiou N, Bates BT, James SL. Asynchrony between subtalar and knee joint function during running. Med Sci Sports Exerc 1999;31(11):1645-55.

35. Mølgaard C, Rathleff MS, Simonsen O. Patellofemoral pain syndrome and its association with hip, ankle, and foot function in 16- to 18-year-old high school students: a single-blind casecontrol study. J Am Podiatr Med Assoc 2011;101(3):215-22.

36. Powers CM, Perry J, Hsu A, Hislop HJ. Are patellofemoral pain and quadriceps femoris muscle torque associated with locomotor function? Phys Ther 1997;77(10):1063-75; discussion 1075-8. 
37. Callaghan MJ, Oldham JA. Quadriceps atrophy: to what extent does it exist in patellofemoral pain syndrome? Br J Sports Med 2004;38(3):295-9.

38. Duvigneaud N, Bernard E, Stevens V, Witvrouw E, Van Tiggelen D. Isokinetic assessment of patellofemoral pain syndrome: a prospective study in female recruits. Isokinet Exerc Sci 2008;16(4):213-9.

39. Boling MC, Padua DA, Marshall SW, Guskiewicz K, Pyne S, Beutler A. A prospective investigation of biomechanical risk factors for patellofemoral pain syndrome: the Joint Undertaking to Monitor and Prevent ACL Injury (JUMP-ACL) cohort. Am J Sports Med 2009;37(11):2108-16.

40. Willson JD, Davis IS. Lower extremity strength and mechanics during jumping in women with patellofemoral pain. J Sport Rehabil 2009;18(1):76-90.

41. Rathleff CR, Baird WN, Olesen JL, Roos EM, Rasmussen S, Rathleff MS. Hip and knee strength is not affected in 12-16 year old adolescents with patellofemoral pain--a cross-sectional population-based study. PLoS One 2013;8(11):e79153.

42. Besier TF, Fredericson M, Gold GE, Beaupré GS, Delp SL. Knee muscle forces during walking and running in patellofemoral pain patients and pain-free controls. J Biomech 2009;42(7):898-905.

43. Aagaard P, Simonsen EB, Trolle M, Bangsbo J, Klausen K. Isokinetic hamstring/quadriceps strength ratio: influence from joint angular velocity, gravity correction and contraction mode. Acta Physiol Scand 1995;154(4):421-7.

44. Li RC, Maffulli N, Hsu YC, Chan KM. Isokinetic strength of the quadriceps and hamstrings and functional ability of anterior cruciate deficient knees in recreational athletes. Br J Sports Med 1996;30(2):161-4.

45. Aagaard P, Simonsen EB, Beyer N, Larsson B, Magnusson P, Kjaer M. Isokinetic muscle strength and capacity for muscular knee joint stabilization in elite sailors. Int J Sports Med 1997;18(7):521-5.

46. Lund-Hanssen H, Gannon J, Engebretsen L, Holen K, Hammer S. Isokinetic muscle performance in healthy female handball players and players with a unilateral anterior cruciate ligament reconstruction. Scand J Med Sci Sports 1996;6(3):1725.

47. Aagaard P, Simonsen EB, Magnusson SP, Larsson B, DyhrePoulsen P. A new concept for isokinetic hamstring: quadriceps muscle strength ratio. Am J Sports Med 1998;26(2):231-7.

48. Jenkins ND, Hawkey MJ, Costa PB, Fiddler RE, Thompson BJ, Ryan ED, et al. Functional hamstrings: quadriceps ratios in elite women's soccer players. J Sports Sci 2013;31(6):612-7.

49. Werner S. Anterior knee pain: an update of physical therapy. Knee Surg Sports Traumatol Arthrosc 2014;22(10):2286-94

50. Westing SH, Seger JY, Karlson E, Ekblom B. Eccentric and concentric torque-velocity characteristics of the quadriceps femoris in man. Eur J Appl Physiol Occup Physiol 1988;58(12):100-4. 